\title{
Hick Samuelson Keynes dynamic economic model with discrete time and consumer sentiment
}

\author{
Loretti I. Dobrescu*, Mihaela Neamtu†, Dumitru Oprisł \\ * University of Venice, Department of Economics, 873 Cannaregio, Venice, 30121 \\ Italy , † West University of Timisoara, Department of Economic Informatics, \\ Mathematics and Statistics, Pestalozzi Street, nr. 16A, Timisoara, 300115 \\ Romania, $\ddagger$ West University of Timisoara, Department of Applied Mathematics, \\ Bd. V. Parvan, nr. 4, Timisoara, 300223 Romania \\ E-mail: lorettiisabella.dobrescu@unipd.it, mihaela.neamtu@fse.uvt.ro, \\ opris@math.uvt.ro
}

Version: March 06, 2009

\begin{abstract}
The paper describes the Hick Samuelson Keynes dynamical economic model with discrete time and consumer sentiment. We seek to demonstrate that consumer sentiment may create fluctuations in the economical activities. The model possesses a flip bifurcation and a Neimark-Sacker bifurcation, after which the stable state is replaced by a (quasi-) periodic motion.
\end{abstract}

Key Words: consumer sentiment, Hick Samuelson Keynes models, NeimarkSacker, flip bifurcation, Lyapunov exponent.

\section{INTRODUCTION}

The economic empirical evidence ([1], [5], [9]) suggests that consumer sentiment influences household expenditure and thus confirms Keynes' assumption that consumer "attitudes" and "animalic spirit" may cause fluctuations in the economic activity. On the other hand, Dobrescu et all. ([3], [4]) analyzed the bifurcation aspects in a discrete-delay Kaldor model of business cycle, which corresponds to a system of equations with discrete time and delay. Following these studies, we develop a dynamic economic model in which the agents' consumption expenditures depend on their sentiment. As particular cases, the model contains: the HickSamulson ([8]), Puu ([8]) and Keynes ([10]) models, as well as the models from [2] and [10]. The model possesses a flip and Neimark-Sacker bifurcation, if the autonomous consumption is variable.

The paper is organized as follows. In Section 2, we describe the dynamic model with discrete time using investment, consumption, sentiment and saving functions. For different values of the model parameters we obtain well-known dynamic models. In Section 3, we analyze the behavior of the dynamic system in the fixed point's neighborhood for the associated map. We establish asymptotic stability conditions for the flip and Neimark-Sacker bifurcations. In both the cases of flip and NeimarkSacker bifurcations, the normal forms are described in Section 4. Using the QR method, the algorithm for determining the Lyapunov exponents is presented in Section 5. Finally, the numerical simulation is done. The analysis of the present model proves its complexity and allows the description of the different scenarios which depend on autonomous consumption. 


\section{THE MATHEMATICAL MODEL WITH DISCRETE TIME AND CONSUMER SENTIMENT}

Let $y_{t}=y(t), t \in N$ be the income at time step $t$ and let:

1. the investment function $I_{t}=I(t), t=2 . . N$, be given by:

$$
I_{t}=v\left(y_{t-1}-y_{t-2}\right)-w\left(y_{t-1}-y_{t-2}\right)^{3}, \quad v>0, w \geq 0 ;
$$

2. the consumption function $C_{t}=C(t), t=2 . . N$, be given by:

$$
C_{t}=a+y_{t-1}\left(b+c S_{t-1}\right), \quad a \geq 0, b>0, c \geq 0,
$$

where $S_{t}=S(t), t=2 . . N$, is the sentiment function given by:

$$
S_{t}=\frac{1}{1+\exp \left(y_{t-1}-y_{t}\right)}, \quad \varepsilon \in[0,1] ;
$$

3. the saving function $E_{t}=E(t), t=2 . . N$, be given by:

$$
E_{t}=d\left(y_{t-2}-y_{t-1}\right)+m S_{t-1}, \quad d \geq 0, m \geq 0 .
$$

The mathematical model is described by the relation:

$$
y_{t}=I_{t}+C_{t}+E_{t} .
$$

From (1), (2), (3), (4) and (5) the mathematical model with discrete time and consumer sentiment is given by:

$y_{t}=a+d y_{t-2}+(b-d) y_{t-1}+v\left(y_{t-1}-y_{t-2}\right)-w\left(y_{t-1}-y_{t-2}\right)^{3}+\frac{c y_{t}+m}{1+\varepsilon \exp \left(y_{t-1}-y_{t}\right)}, t=2 . . N$.

The parameters from (6) have the following economic interpretations. The parameter $a$ represents the autonomous expenditures. The parameter $d$ is the control, $d \in[0,1]$ and it characterizes a part of the difference between the incomes obtained at two time steps $t-2$ and $t-1$, which is used for consumption or saving in the time step $t$. The parameter $c, c \in[0,1]$, is the trend towards consumption. The parameter $m, m \in[0,1]$, is the trend towards the saving. The parameter $b$, $b \in(0,1)$, represents the consumer's reaction against the increase or decrease of his income. When the income (strongly) decreases, the consumer becomes pessimistic and consumes $0<b<1$ of his income. When the income (strongly) increases, the consumer becomes optimistic and consumes $b<b+c<1$ of his income. Note that Souleles ([8]) finds, in fact, that higher consumer confidence is correlated with less saving and increases in relation to expected future resources. The parameters $v$ and $w, v>0, w \geq 0$ describe the investment function. If $w=0$ the investment function is linear. The parameter $\varepsilon, \varepsilon \in[0,1]$, describes a family of the sentiment functions.

For different values of the model parameters, we obtain the following classical models:

1. for $a=0, b=1-s, d=0, m=0, \varepsilon=0, s \in(0,1)$ from (6) we obtain the Hick-Samuelson model ([7]):

$$
y_{t}=(1+v-s) y_{t-1}-v y_{t-2}, \quad v>0, s \in(0,1)
$$


2. for $v=w=0, \varepsilon=0, m=0$, (6) gives us the Keynes model ([7]):

$$
y_{t}=d\left(y_{t-2}-y_{t-1}\right)+a+b y_{t-1}
$$

3. for $v=w=0, \varepsilon=1$, (6) leads to the model from [2]:

$$
y_{t}=d\left(y_{t-2}-y_{t-1}\right)+a+b y_{t-1}+\frac{c y_{t-1}+m}{1+\exp \left(y_{t-2}-y_{t-1}\right)}, t \in N ;
$$

4. for $a=0, b=s-1, d=0, m=0, w=1+v, \varepsilon=0$, from (6) we get the Puu model ([7]):

$$
y_{t}=v\left(y_{t-1}-y_{t-2}\right)-(1+v)\left(y_{t-1}-y_{t-2}\right)^{3}-(1-s) y_{t-1} .
$$

\section{THE DYNAMIC BEHAVIOR OF THE MODEL (6)}

Using the method from Kusnetsov [6] and [3] and [4], we will analyze the system (6), considering the parameter a as bifurcation parameter. The associated map of (6) is $F: \mathbb{R}^{2} \rightarrow \mathbb{R}^{2}$ given by:

$$
F\left(\begin{array}{l}
y \\
x
\end{array}\right)=\left(\begin{array}{c}
a+(b-d+v) y+(d-v) z-w(y-z)^{3}+\frac{c y+m}{1+\varepsilon \exp (z-y)} \\
y
\end{array}\right)
$$

Using the methods from [3], [4] and [6], the map (7) has the following properties:

Proposition 1. (i). If $(1+\varepsilon)(1-b)-c>0$, then, for the map (7), the fixed point with the positive components is $E_{0}\left(y_{0}, z_{0}\right)$, where:

$$
y_{0}=p_{1} a+p_{2} ; \quad z_{0}=y_{0}
$$

and

$$
p_{1}=\frac{1+\varepsilon}{(1+\varepsilon)(1-b)-c}, p_{2}=\frac{m}{(1+\varepsilon)(1-b)-c} ;
$$

(ii). The Jacobi matrix of the map $F$ in $E_{0}$ is given by:

$$
A=\left(\begin{array}{cc}
a_{11} & a_{12} \\
1 & 0
\end{array}\right),
$$

where: $a_{11}=p_{3} a-p_{4}+p_{5}, a_{12}=-p_{3} a+p_{4}$,

$p_{3}=\frac{\varepsilon c}{(1+\varepsilon)^{2}} p_{1}, p_{4}=d-v-\frac{\varepsilon m}{(1+\varepsilon)^{2}}-\frac{\varepsilon c}{(1+\varepsilon)^{2}} p_{2}, p_{5}=b+\frac{c}{(1+\varepsilon)}$;

(iii). The characteristic equation of matrix $A$ is given by:

$$
\lambda^{2}-a_{11} \lambda-a_{12}=0 ;
$$

(iv). If the model parameters $d, v, \varepsilon, b, c, m$ satisfy the following inequality:

$$
(1+d-v)(1+\varepsilon)((1+\varepsilon)(1-b)-c)-m \varepsilon(1-b)>0,
$$

then, for equation (10), the roots have the modulus less than 1 , if and only if $a \in\left(a_{1}, a_{2}\right)$, where

$$
a_{1}=\frac{2 p_{4}-p_{5}-1}{2 p_{3}}, a_{2}=\frac{1+p_{4}}{p_{3}}
$$

(v). If the model parameters $d, v, \varepsilon, b, c, m$ satisfy the inequality (11) and $a=a_{1}$ then, one of equation (10)'s roots is -1 , while the other one has the modulus less than 1;

(vi) If the model parameters $d, v, \varepsilon, b, c, m$ satisfy the inequality (11) and $a=a_{2}$ then, the equation (10) has the roots $\mu_{1}(a)=\mu(a), \mu_{2}(a)=\bar{\mu}(a)$, where $|\mu(a)|=1$. 
Using [7] and Proposition 1, with respect to parameter $a$, the asymptotic stability conditions of the fixed point, the conditions for the existence of the flip and Neimark-Sacker bifurcations are presented in:

Proposition 2. (i). If $(1+\varepsilon)(1-b)-c>0$, the inequality (11) holds and $2 p_{4}-p_{5}-1>0$, then for $a \in\left(a_{1}, a_{2}\right)$ the fixed point $E_{0}$ is asymptotically stable. If $(1+\varepsilon)(1-b)-c>0$, the inequality (11) holds and $2 p_{4}-p_{5}-1<0$, then for $a \in\left(0, a_{2}\right)$ the fixed point $E_{0}$ is asymptotically stable;

(ii). If $(1+\varepsilon)(1-b)-c>0$, the inequality (11) holds and $2 p_{4}-p_{5}-1>0$, then $a=a_{1}$ is a flip bifurcation and $a=a_{2}$ is a Neimark-Sacker bifurcation;

(iii). If $(1+\varepsilon)(1-b)-c>0$, the inequality (11) holds and $2 p_{4}-p_{5}-1<0$, then $a=a_{2}$ is a Neimark-Sacker bifurcation.

\section{THE NORMAL FORM FOR FLIP AND NEIMARK-SACKER BIFURCATIONS}

In this section, we describe the normal form in the neighborhood of the fixed point $E_{0}$, for the cases $a=a_{1}$ and $a=a_{2}$.

We consider the transformation:

$$
u_{1}=y-y_{0}, \quad u_{2}=z-z_{0}
$$

where $y_{0}, z_{0}$ are given by (8). With respect to (13), the map (7) is $G: \mathbb{R}^{2} \rightarrow \mathbb{R}^{2}$, where

$$
G\left(u_{1}, u_{2}\right)=\left(\begin{array}{l}
g_{1}\left(u_{1}, u_{2}\right) \\
g_{2}\left(u_{1}, u_{2}\right)
\end{array}\right)
$$

and

$$
\begin{aligned}
& g_{1}\left(u_{1}, u_{2}\right)=-\frac{r}{1+\varepsilon}+(b-d+v) u_{1}+(d-v) u_{2}-w\left(u_{1}-u_{2}\right)^{3}+\frac{c u_{1}+r}{1+\varepsilon \exp \left(u_{2}-u_{1}\right)} \\
& g_{2}\left(u_{1}, u_{2}\right)=u_{1}, r=c y_{0}+m
\end{aligned}
$$

The map (14) has $O(0,0)$ as fixed point.

We consider:

$$
\begin{aligned}
a_{11} & =\frac{\partial g_{1}}{\partial u_{1}}(0,0), a_{12}=\frac{\partial g_{1}}{\partial u_{2}}(0,0), l_{20}=\frac{\partial^{2} g_{1}}{\partial u_{1}^{2}}(0,0), l_{11}=\frac{\partial^{2} g_{1}}{\partial u_{1} \partial u_{2}}(0,0) \\
l_{02}= & \frac{\partial^{2} g_{1}}{\partial u_{2}^{2}}(0,0), l_{30}=\frac{\partial^{3} g_{1}}{\partial u_{1}^{3}}(0,0), l_{21}=\frac{\partial^{3} g_{1}}{\partial u_{1}^{2} \partial u_{2}}(0,0), l_{12}=\frac{\partial^{3} g_{1}}{\partial u_{1} \partial u_{2}^{2}}(0,0), l_{03}=\frac{\partial^{3} g_{1}}{\partial u_{1}^{3}}(0,0)
\end{aligned}
$$

We develop the function $G(u), u=\left(u_{1}, u_{2}\right)^{T}$ in the Taylor series until the third order and obtain:

$$
G(u)=A u+\frac{1}{2} B(u, u)+\frac{1}{2} C(u, u, u)+O\left(|u|^{4}\right)
$$

where $A$ is given by (9) and

$$
B(u, u)=\left(B^{1}(u, u), 0\right)^{T}, C(u, u, u)=\left(C^{1}(u, u, u), 0\right)^{T}
$$




$$
\begin{aligned}
B^{1}(u, u) & =u^{T}\left(\begin{array}{ll}
l_{20} & l_{11} \\
l_{11} & l_{02}
\end{array}\right) u, \\
C^{1}(u, u, u) & =u^{T}\left(u_{1}\left(\begin{array}{ll}
l_{30} & l_{21} \\
l_{21} & l_{12}
\end{array}\right)+u_{2}\left(\begin{array}{ll}
l_{21} & l_{12} \\
l_{12} & l_{03}
\end{array}\right)\right) u .
\end{aligned}
$$

For $a=a_{1}$, given by (12) with the condition $(v)$ from Proposition 1, we have:

Proposition 3. (i). The eigenvector $q \in \mathbb{R}^{2}$, given by $A q=-q$ has the components:

$$
q_{1}=1, q_{2}=-1 .
$$

(ii) The eigenvector $h \in \mathbb{R}^{2}$, given by $h^{T} A=-h^{T}$ has the components:

$$
h_{1}=\frac{1}{1+a_{12}}, h_{2}=-\frac{a_{12}}{1+a_{12}} .
$$

The relation $\langle q, h\rangle=1$ holds.

(iii). The normal form of the map (7) on the center manifold in $O(0,0)$ is given by:

$$
\eta \rightarrow-\eta+\frac{1}{6} \nu \eta^{3}+O\left(\eta^{4}\right),
$$

where $\nu=\frac{1}{1+a_{12}}\left(l_{30}-3 l_{21}+3 l_{12}-l_{03}\right)+\frac{3}{1-a_{11}-a_{12}}\left(l_{20}-2 l_{11}+l_{02}\right)^{2}$.

The proof results from straight calculus using the formula (6):

$$
\nu=<r, C(q, q, q)>+3 B\left(q,(I-A)^{-1} B(q, q)\right), I=\left(\begin{array}{ll}
1 & 0 \\
0 & 1
\end{array}\right) .
$$

For $a=a_{2}$, given by (12) with the condition (vi) from Proposition 1, we have:

Proposition 4. (i). The eigenvector $q \in \mathbb{C}^{2}$, given by $A q=\mu_{1} q$, where $\mu_{1}$ is the eigenvalue of the matrix $A$, has the components:

$$
q_{1}=1, q_{2}=\mu_{2}=\bar{\mu}_{1} .
$$

(ii) The eigenvector $h \in \mathbb{C}^{2}$, given by $h^{T} A=\mu_{2} h^{T}$, where $\mu_{2}=\bar{\mu}_{1}$, has the components:

$$
h_{1}=\frac{1}{1+\mu_{1}^{2} a_{12}}, h_{2}=\frac{\mu_{1} a_{12}}{1+\mu_{1}^{2} a_{12}} .
$$

The relation $\langle q, \bar{h}\rangle=1$ holds.

Using (15) and (16) we have:

$$
\begin{aligned}
& B^{1}(q, q)=l_{20}+2 l_{11} \mu_{2}+l_{02} \mu_{2}^{2}, \\
& B^{1}(q, \bar{q})=l_{20}+l_{11}\left(\mu_{1}+\mu_{2}\right)+l_{02} \mu_{1} \mu_{2}, \\
& B^{1}(\bar{q}, \bar{q})=l_{20}+2 l_{11} \mu_{1}+l_{02} \mu_{1}^{2} .
\end{aligned}
$$

We denoted by:

$$
\begin{aligned}
& g_{20}=v_{1} B^{1}(q, q), g_{11}=v_{1} B^{1}(q, \bar{q}), g_{02}=v_{1} B^{1}(\bar{q}, \bar{q}), \\
& h_{20}^{1}=\left(1-v_{1}-\bar{v}_{1}\right) g_{20}, h_{11}^{1}=\left(1-v_{1}-\bar{v}_{1}\right) g_{11}, h_{02}^{1}=\left(1-v_{1}-\bar{v}_{1}\right) g_{02}, \\
& h_{20}^{2}=-\left(v_{1} \mu_{2}+\bar{v}_{1} \mu_{1}\right) B^{1}(q, q), h_{11}^{2}=-\left(v_{1} \mu_{2}+\bar{v}_{1} \mu_{1}\right) B^{1}(q, \bar{q}), \\
& h_{02}^{2}=-\left(v_{1} \mu_{2}+\bar{v}_{1} \mu_{1}\right) B^{1}(\bar{q}, \bar{q}),
\end{aligned}
$$




$$
w_{20}=\left(\mu_{1}^{2} I-A\right)^{-1}\left(\begin{array}{c}
h_{20}^{1} \\
h_{20}^{2}
\end{array}\right), w_{11}=(I-A)^{-1}\left(\begin{array}{c}
h_{11}^{1} \\
h_{11}^{2}
\end{array}\right), w_{02}=\left(\mu_{2}^{2}-A\right)^{-1}\left(\begin{array}{c}
h_{02}^{1} \\
h_{02}^{2}
\end{array}\right),
$$

where $I=\left(\begin{array}{ll}1 & 0 \\ 0 & 1\end{array}\right), A$ is given by (9) and

$$
\begin{aligned}
r_{20} & =B^{1}\left(q, w_{20}\right), r_{11}=B^{1}\left(q, w_{11}\right), \\
C_{0} & =C^{1}(q, q, \bar{q})=l_{30}+\left(\mu_{1}+2 \mu_{2}\right) l_{21}+\mu_{2}\left(2 \mu_{1}+\mu_{2}\right) l_{12}+\mu_{1} \mu_{2}^{2} l_{03}, \\
g_{21} & =v_{2}\left(r_{20}+2 r_{11}+C_{0}\right) .
\end{aligned}
$$

Using the normal form for the Neimark-Sacker bifurcation of the dynamic systems with discrete time [6] and (17), (18), (19), we obtain:

Proposition 5. (i). The solution of the system (6) in the neighborhood of the fixed point $\left(y_{0}, z_{0}\right) \in \mathbb{R}^{n}$ is given by:

$$
\begin{aligned}
y_{t} & =y_{0}+\mu_{2} x_{t}+\mu_{1} \bar{x}_{t}+\frac{1}{2} w_{20}^{2} x_{t}^{2}+w_{11}^{2} x_{t} \bar{x}_{t}+\frac{1}{2} w_{02}^{2} \bar{x}_{t}^{2}, \\
y_{t-1} & =z_{t}=z_{0}+x_{t}+\bar{x}_{t}+\frac{1}{2} w_{20}^{1} x_{t}^{2}+w_{11}^{1} x_{t} \bar{x}_{t}+\frac{1}{2} w_{02}^{1} \bar{x}_{t}^{2},
\end{aligned}
$$

where $x_{t}$ is the solution of the following equation:

$$
x_{t+1}=\mu x_{t}+\frac{1}{2} g_{20} x_{t}^{2}+g_{11} x_{t} \bar{x}_{t}+\frac{1}{2} g_{02} \bar{x}_{t}^{2}+\frac{1}{2} g_{21} x_{t}^{2} \bar{x}_{t} .
$$

(ii) A complex variable transformation exists so that the equation (20) becomes:

$$
\omega_{t+1}=\mu_{1} \omega_{t}+C^{1} \omega_{t}^{2} \bar{\omega}_{t}+O\left(\left|\omega_{t}\right|^{4}\right),
$$

where

$$
C^{1}=\frac{g_{20} g_{11}\left(\mu_{2}-3-2 \mu_{1}\right)}{2\left(\mu_{1}^{2}-\mu_{1}\right)\left(\mu_{2}-1\right)}+\frac{\left|g_{11}\right|^{2}}{\left(1-\mu_{1}\right)}+\frac{\left|g_{20}\right|^{2}}{2\left(\mu_{1}^{2}-\mu_{2}\right)}+\frac{g_{21}}{2} .
$$

(iii). If $l_{0}=\operatorname{Re}\left(e^{-i \theta} C^{1}\right)<0$, where $\theta=\arg \left(\mu_{1}\right)$, then in the neighborhood of the fixed point $\left(y_{0}, z_{0}\right)$ there is a stable limit cycle.

\section{THE LYAPUNOV EXPONENTS}

If $a_{1}>0$ then for $a \in\left(0, a_{1}\right)$ or $a \in\left(a_{2}, 1\right)$ the system (6) has a complex behavior and it can be established by computing the Lyapunov exponents. We will use the algorithm QR from [6] and the determination of the Lyapunov exponents can be obtained by solving the following system:

$$
\begin{aligned}
y_{t+1} & =a+(b-d+v) y_{t}+(d-v) z_{t}-w\left(y_{t}-z_{t}\right)^{3}+\frac{c y_{t}+m}{1+\exp \left(z_{t}-y_{t}\right)} \\
z_{t+1} & =y_{t} \\
x_{t+1} & =\arctan \left(-\frac{\cos x_{t}}{f_{11} \cos x_{t}-f_{12} \sin x_{t}}\right) \\
\lambda_{t+1} & =\lambda_{t}+\ln \left(\left|\left(f_{11}-\tan x_{t+1}\right) \cos x_{t} \cos x_{t+1}-f_{12} \sin x_{t} \cos x_{t+1}\right|\right) \\
\mu_{t+1} & =\mu_{t}+\ln \left(\left|\left(f_{11}-\tan x_{t+1}+1\right) \sin x_{t} \cos x_{t+1}+f_{12} \cos x_{t} \cos x_{t+1}\right|\right),
\end{aligned}
$$


with

$$
\begin{aligned}
& f_{11}=\frac{\partial f_{1}}{\partial y}\left(y_{t}, z_{t}\right)=b-d+v-3 w\left(y_{t}-z_{t}\right)^{2}+\frac{c+\varepsilon\left(c+m+c y_{t}\right) \exp \left(z_{t}-y_{t}\right)}{\left(1+\varepsilon \exp \left(z_{t}-y_{t}\right)\right)^{2}}, \\
& f_{12}=\frac{\partial f_{1}}{\partial z}\left(y_{t}, z_{t}\right)=d-v+3 w\left(y_{t}-z_{t}\right)^{2}-\frac{\varepsilon\left(m+c y_{t}\right) \exp \left(z_{t}-y_{t}\right)}{\left(1+\varepsilon \exp \left(z_{t}-y_{t}\right)\right)^{2}} .
\end{aligned}
$$

The Lyapunov exponents are:

$$
L_{1}=\lim _{t \rightarrow \infty} \frac{\lambda_{t}}{t}, L_{2}=\lim _{t \rightarrow \infty} \frac{\mu_{t}}{t}
$$

If one of the two exponents is positive, the system has a chaotic behavior.

\section{NUMERICAL SIMULATION}

The numerical simulation is done using a Maple 11 program. We consider different values for the parameters which are used in the real economic processes. The bifurcation parameter is $a$.

For $a=250, v=0,1, w=0, c=0,1, b=0,45, \varepsilon=1, m=0,5$ and $d=0$ we obtain Fig1, while for $a=250, v=0,1, w=0, c=0,1, b=0,45, \varepsilon=1, m=0,5$ and $d=0,6$ we obtain Fig2. Both figures represent the income $y_{t}$ with respect to the income $y_{t-1}$. In both these cases, the system is not chaotic.
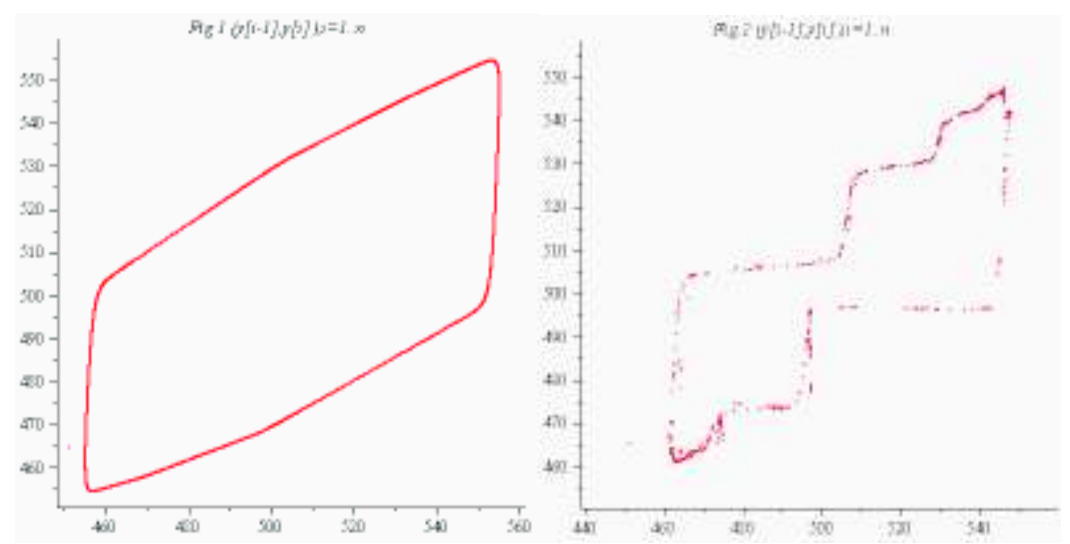

For $a=250, v=0,1, w=0, c=0,1, b=0,45, \varepsilon=1, m=0,5$ and $d=0,8$ we obtain Fig3 and Fig4, which represent the income $y_{t}$ with respect to the income $y_{t-1}$ and the Lyapunov exponent. In these cases, the system has a chaotic behavior. 


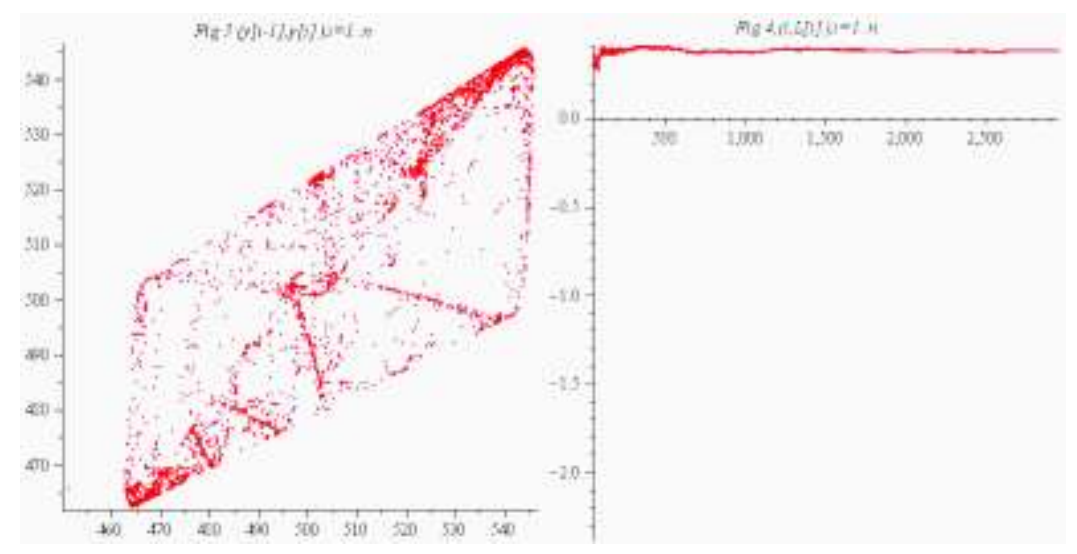

For different values of the parameters, we can obtain a Neimark-Sacker bifurcation point or a flip bifurcation point and a Neimark-Sacker bifurcation point.

\section{REFERENCES}

[1] Carroll, C., Fuhrer, J., Wilcox, D., 'Does consumer sentiment forecast household spending? If so, why?', American Economic Review, 84, 1397-1408, 1994.

[2] Chis, O., Oprisan, O., Opris, D., 'Keynes dynamic model of savings with behavior function', The Proceeding of the Faculty of Economics, EuroUniversity Dragan, Lugoj, Romania 2008 (in press).

[3] Dobrescu, L.I., Opris, D., 'Neimark-Sacker bifurcation for the discrete-delay Kaldor model', Chaos, Solitons \& Fractals, doi:10.1016/j.chaos.2007.10.044, 2007.

[4] Dobrescu, L.I., Opris, D., 'Neimark-Sacker bifurcation for the discrete-delay Kaldor-Kalecki model', Chaos, Solitons \& Fractals, doi:10.1016/j.chaos.2008.09.022, 2008.

[5] Doms, M., Morin, N., 'Consumer sentiment, the economy, and the news media', Federal Reserve Bank of San Francisco, Working Paper 2004-09.

[6] Kuznetsov, Y.A., 'Elements of Applied Bifurcation Theory', Applied Mathematical Sciences 112, Springer-Verlag, New York, 1995.

[7] Neamtu, M., Opris, D., 'Economical games. Disctrete economical dynamics. Applications', Mirton, 2008 (in Romanian).

[8] Puu, T., Sushko, I., 'A business cycle model with cubic nonlinearity', Chaos, Solitons and Fractals 19, 597-612, 2004.

[9] Souleles, N., 'Expectations, heterogeneous forecast errors, and consumption: Micro evidence from the Michigan Consumer Sentiment Survey', Journal of Money, Credit and Banking, 36, 39-72, 2004.

[10] Westerhoff, F.H., 'Consumer sentiment and business cycles: A Neimark-Sacker bifurcation scenario', Applied Economics Letters, 14, 1-5, 2007. 